\title{
BMJ Open Platelet function and cardiovascular risk in adult HIV-infected patients on HAART: a protocol for a systematic review and meta-analysis
}

\author{
Bongani Brian Nkambule, ${ }^{1}$ Zibusiso Mkandla, ${ }^{1}$ Tinashe Mutize, ${ }^{1}$ \\ Phiwayinkosi Vusi Dludla ${ }^{2}$
}

To cite: Nkambule BB, Mkandla Z, Mutize T, et al. Platelet function and cardiovascular risk in adult HIVinfected patients on HAART: a protocol for a systematic review and meta-analysis. BMJ Open 2017;7:e019468. doi:10.1136/ bmjopen-2017-019468

- Prepublication history and additional material for this paper are available online. To view these files, please visit the journal online (http://dx.doi. org/10.1136/bmjopen-2017019468).

Received 4 September 2017 Revised 26 0ctober 2017 Accepted 31 October 2017

\section{CrossMark}

${ }^{1}$ School of Laboratory Medicine and Medical Sciences, College of Health Sciences, University of KwaZulu-Natal, Durban, South Africa

${ }^{2}$ Biomedical Research and Innovation Platform, Medical Research Council, Tygerberg, South Africa

Correspondence to Dr Bongani Brian Nkambule; nkambuleb@ukzn.ac.za

\section{ABSTRACT}

Introduction The incidence of cardiovascular disease (CVD) is now at least threefold higher in HIV-infected patients as compared with the general population. Although platelet activation and reactivity are implicated in the development of CVDs in HIV-infected patients, its precise role remains inconclusive. We aim to assess the association between platelet activation and selected cardiovascular risk factors in HIV-1-infected individuals on highly active antiretroviral treatment (HAART).

Methods This will be a systematic review and meta-analysis of published studies evaluating the association between platelet activation and CVD risk factors in HAART-treated adults. The search strategy will include medical subject headings words for MEDLINE, and this will be adapted to Embase search headings (Emtree) terms for the EMBASE database. The search will cover literature published between 1 January 1996 to 30 April 2017. Studies will be independently screened by two reviewers using predefined criteria. Relevant eligible full texts will be screened; data will be extracted, and a qualitative synthesis will be conducted. Data extraction will be performed using Review Manager V.5.3. To assess the quality and strengths of evidence across selected studies, the Grading of Recommendations Assessment Development and Evaluation approach will be used. The Cochran's $Q$ statistic and the $I^{2}$ statistics will be used to analyse statistical heterogeneity between studies. If included studies show high levels of homogeneity, a random effects meta-analysis will be performed using $R$ statistical software.

Ethics and dissemination This will be a review of existing studies and will not require ethical approval. The findings will be disseminated through peer-reviewed publication and presented at local and international conferences. An emerging patient management dilemma is that of the increased incidence of CVD in people living with HIV on HAART. This review may inform treatment and cardiovascular risk stratification of HIV-infected patients at increased risk of developing CVD.

PROSPERO registration number CRD42017062393.

\section{INTRODUCTION}

The incidence of cardiovascular disease (CVD) in HIV-infected patients is on the
Strengths and limitations of this study

- This review will comprehensively evaluate published literature on the primary research question, focusing on platelet function in HIV-infected individuals on highly active antiretroviral treatment (HAART) and the risk of cardiovascular disease.

- A comprehensive search strategy will be used to identify and retrieve articles relevant to our research question.

- This will be the first systematic review and metaanalysis aimed at addressing the controversial topic of platelet function in people living with HIV on HAART.

- The main limitation will be the heterogeneity of the studies available in terms of clinical, methodological, study designs and outcome definitions.

rise in both developing and developed countries. ${ }^{12}$ The (HIV) pandemic continues to devastate resource-poor and developing countries. Globally, an estimated number of 34.5 million adults are living with HIV. ${ }^{3}$ The East and Southern African regions have the highest number of people living with HIV (PLWH), with an estimated total of 6.3 million PLWH. ${ }^{4}$ South Africa represents a quarter of the burden of HIV in sub-Saharan Africa with an estimated antiretroviral (ARV) coverage of $50.95 \% .^{4}$ Early initiation of highly active antiretroviral therapy (HAART) will ultimately commit HIV-infected individuals to prolonged antiretroviral drug exposure. The initiation of HAART is associated with an increased risk of CVD. Whereby, HAART induces dyslipidaemia ${ }^{5}$ obesity, ${ }^{6}$ increased platelet reactivity ${ }^{78}$ and ultimately increases the risk of developing CVD. ${ }^{8-12}$

HAART is based on protease inhibitors (PIs), which have been shown to exacerbate inflammation, ${ }^{13}$ induce insulin 
resistance, ${ }^{9}{ }^{14}$ alter platelet function (reviewed in ref 11) and increase the risk of CVD. ${ }^{12}$ PIs induce insulin resistance by altering lipid metabolism resulting in increased fasting triglycerides and free fatty acids. In addition, certain PIs interfere with the glucose transporter type 4-mediated glucose transport. ${ }^{14} 15$ A steady increase in the incidence of metabolic syndrome has been reported in PLWH. ${ }^{16}$ An emerging patient management dilemma is that of the increased incidence of type 2 diabetes on the onset of HAART. ${ }^{17-19}$ In fact, a fourfold increase in the prevalence of type 2 diabetes has been reported in HIV-infected patients on HAART. ${ }^{20}$ In the large data collection on adverse events of anti-HIV drugs (D: A: D) cohort, HAART was associated with increased risk of type 2 diabetes. ${ }^{21}$

Both chronic inflammation and immune activation have been identified as key driving forces in the pathogenesis of non-AIDS-related deaths. ${ }^{1}$ Platelets play a role in linking inflammation, chronic immune activation and microvascular dysfunction. ${ }^{22}$ The platelet reactivity index is a robust measure of the ratio of platelet aggregates in peripheral blood, and ratios above 1.05 have been associated with pathological platelet aggregation. ${ }^{23}$ The role of platelet activation and increased risk of CVD in HIV-infected patients has been described. ${ }^{84}$ However, contradictory findings on the activation status of platelets and the risk of CVD in HIV-infected patients have been reported, ${ }^{25}$ and a clear understanding of the underlying mechanism remains scarce.

The benefits of early treatment interventions are well described. However, analysis of data from the large Strategies for the Management of Antiretroviral Therapy Study showed that markers of inflammation correlated with adverse outcomes in patients on antiretroviral regimens. ${ }^{26}$ Moreover, knowledge gained from studies conducted on simian immunodeficiency virus (simian equivalent of HIV) hosts suggests that chronic immune activation is a key contributor to the pathogenic infection. ${ }^{27}$ Therefore, despite adequate control of viral loads in first-world countries, an era of non-AIDS-related deaths, such as myocardial infarctions and cerebrovascular accidents (CVAs), now prevails. ${ }^{28}$ Furthermore, studies have shown that levels of activated $\mathrm{B}$ and $\mathrm{T}$ cell lymphocytes remain high in HIV-infected individuals despite successful antiretroviral therapy (ART).$^{29}$ In addition, an increase in activated CD8+ T cells after 6 months of suppressive ART is associated with a 1.6- fold increased risk of subsequent death. ${ }^{30}$ Persistent T cell activation induces increased $\mathrm{CD}^{+} \mathrm{T}$ cell turn over, which may result in the exhaustion of the immune system and alterations in the regenerative capacity of immune cells. ${ }^{31}$ To date, there are currently no published systematic reviews providing cumulative evidence on platelet activation and reactivity in patients with HIV and the risk of CVDs. This systematic review will aim to assess the available evidence of the association between platelet function (activation and reactivity) and cardiovascular risk in HIV-1-infected individuals on HAART.

\section{Research question}

1. Are activated platelets and their reactivity index associated with cardiovascular risk factors (hypertension, diabetes, dyslipidaemia, metabolic syndrome, coagulation factors) in HIV-infected patients on HAART?

\section{Objectives}

1. To estimate the overall measures of effect for the association between activated platelets and HIV infection in patients on HAART compared with untreated and uninfected individuals.

2. To estimate the overall measures of effect for the association between platelet activation and cardiovascular risk in HIV-infected patients on HAART.

3. To estimate the overall effect for the association of platelet hyper-reactivity and cardiovascular risk in HIV-infected patients on HAART.

\section{METHODS}

The systematic review protocol has been prepared according to the Preferred Reporting Items for Systematic Review and Meta-Analysis Protocols 2015 guidelines. ${ }^{32}$ Details pertaining the protocol for this systematic review were registered on PROSPERO (PROSPERO number: CRD42017062393) and can be accessed online (https://www.crd.york.ac.uk/PROSPERO/display_ record.asp?ID=CRD42017062393).

\section{Eligibility criteria}

Study design

All relevant cross-sectional and case control studies with a clearly defined control population will be included. In addition, randomised controlled trials (RCTs) and retrospective and prospective cohort studies with defined time points highlighting data points before and after intervention will be included. Case studies and case reports will be excluded from the review.

\section{Participants}

Studies evaluating platelet function in HIV-1-infected adults defined as 18 years or older will be included. We will include studies that have reported the baseline HIV-1 viral load and initial CD4 counts and platelet counts of all participants enrolled. In addition, we will also include studies that report the exclusion of participants on non-steroidal anti-inflammatory drugs or aspirin. Studies will be included regardless of the metabolic profile of participants included. Studies that include pregnant women, patients with a known history of malignancy and treatment with anticoagulants will be excluded.

Interventions

We will consider studies that have clearly defined HAART regime of either three or more antiretroviral 
drugs. Furthermore, we will consider other forms of antiplatelet interventions provided the mode of administration and dose is reported. The primary comparisons that will made will include;

\section{Comparators}

Comparators will differ based on the study design and will include participants randomised not to receive antiretroviral intervention and participants who are not HIV infected (case-control studies and cohort studies).

\section{Interventions that affect platelet function}

1. The use of statins in combination to ART and antihyperglycaemic drugs.

2. Differences between the ART drug combinations and timing of interrupted treatment compared with uninterrupted treatment.

\section{Outcomes}

The clinical endpoints important for decision making include:

\section{Primary endpoints}

1. Platelet activation (reported as mean/median percentage platelet P-selectin expression or platelet monocyte aggregates).

2. CVD (proportion of incidents reported as Risk ratio $(\mathrm{RR}))$ :
a. Coronary artery events: fatal myocar- dial infarction, non-fatal myocardial infarction, unstable angina and stable angina.

b. Cerebrovascular events: fatal stroke, non-fatal stroke (ischaemic or haemorrhagic), transient ischaemic attack and vascular events.

3. Type 2 diabetes (OR as a primary effect measure or $\mathrm{HR}$ as a secondary measure).

4. Metabolic syndrome (MetS) (reported as mean percentage of participants with MetS or the RR as an effect measure).

\section{Surrogate outcomes}

1. Cardiovascular risk (total cholesterol, high-density lipoprotein cholesterol level, systolic blood pressure, hypertension treatment, smoking, diabetes, dyslipidaemia and metabolic syndrome).

2. Hyper-reactive platelets (increased platelet aggregation and activation).

3. Increased levels of markers of coagulation (D-dimers, fibrinogen, von Willebrand factor and tissue factor).

4. Insulin resistance and impaired glucose tolerance.

5. Thrombocytopaenia (severe or mild).

All outcomes will be extracted from the included studies and reported. Due to the broad category of CVD, we will extract the type of cardiovascular event reported from each respective study. The surrogate outcomes for type 2 diabetes will include insulin resistance and impaired glucose tolerance that may be reported based on varying outcome measures. The methods and classifications
American Diabetes Association or WHO classification used will also be extracted and reported.

\section{Literature searches}

A search strategy will be developed using medical subject headings words for MEDLINE, and this will be adapted to Embase search headings (Emtree) terms for the Embase database (see online supplementary appendix 1). The search terms will also be applied to retrieve studies using the PubMed clinical queries. The electronic database search will be augmented by searching the Cochrane Central Register of Clinical trials (Wiley interface), the metaregister (www.controlled-trials.com/mrct/), Web of science and Global Index of Medicus. In addition, the reference list of selected studies will be scanned to identify relevant literature. The search strategy will be adapted for syntax used in Embase database. The strategy will be peer reviewed by a liberian specialist.

The systematic search will be conducted without any language restrictions. The literature retrieved will be restricted to human studies. The search strategy will consist of the following major keywords: Platelets; Platelet P-selectin; Platelet CD40L; Platelet monocyte aggregates; Platelet leukocyte aggregates; Platelet monocyte complexes; HIV-1 infection; and diabetes. For each keyword, multiple synonyms will be searched in the title or abstract.

\section{Study records}

Data management

\section{Collection process}

To minimise data entry errors, the web-based EPPI-Reviewer V.4.0 software for research synthesis ${ }^{33}$ will be used for data extraction into predefined data forms and exported directly into statistical software. The Mendeley reference manager (V.1.17.10, Mendeley), will be used to identify duplicates.

\section{Selection process}

Two independent reviewers (BBN and PVD) will conduct the selection procedure. Each reviewer will screen the titles, abstracts and full texts in contrast to the inclusion criteria. The exclusion criteria for title and abstract screening phase will include:

1. Review articles.

2. Animal studies or animal models of HIV infection.

3. Non-HIV-1-related studies.

The additional criteria will be used for screening full text:

1. Full text not available.

2. Duplicate publication of the same study cohort.

3. Published conference abstracts.

\section{Data items}

The following relevant items will be extracted: author, year, original language, sample size, years of follow-up, outcome measures, patient characteristics (gender ratio, metabolic profile, levels of measured coagulation parameters, platelet counts, levels of immune activation or 
inflammation, mean CD4 counts HIV-1 RNA levels, duration of infection, duration and type of antiplatelet drugs used, type of HAART and dosage administered).

In addition, details related to the assays used to measure HIV-1 RNA and platelet function (activation or aggregation) as well as the use of platelet separation techniques (preparation of platelet-rich plasma or platelet poor plasma) will be extracted. In instances where there are no reported data amputation techniques on priority outcomes and when the effect size cannot be calculated, the authors will be contacted for additional data.

\section{Data simplification}

Individual studies may report varying dosage of treatment interventions used. To reduce potential bias that may be introduced by multiple comparisons with a single control group, these will all be grouped into a single drug-specific treatment group.

\section{Risk of bias in individual studies}

To assess the potential risk of bias in RCT, information will be collected in accordance to the Cochrane collaboration tool for assessing bias. ${ }^{34} \mathrm{~A}$ judgement on the possible risk of bias of extracted information will be made based on each of the six domains. For observational studies, the relevant domains will be considered; these will include participant selection, measurement of variables, confounders and missing data. The judgement will be made independently by two reviewers (ZM and PVD) based on the criteria defined for judging the risk of bias. In an instance of disagreements between the two reviewers, BBN will be consulted for arbitration.

\section{Data synthesis}

Assessment of clinical and methodological heterogeneity will be performed first, and this will then be followed by an assessment of statistical heterogeneity. The Cochran's $\mathrm{Q}$ statistic $^{35}$ and the $\mathrm{I}^{2}$ statistics will be used to analyse statistical heterogeneity between studies. ${ }^{36}$ If studies show similarity with regards to (participants, intervention, comparisons, outcomes). If an efficient number of studies included are homogeneous in terms of extracted information, we will conduct a meta-analysis using R statistical Software (The R foundation for statistical computing, Vienna, Austria). An $\mathrm{I}^{2}$ value of $>25$ will be considered as moderate or substantial heterogeneity. The random-effects model will be used should there be significant levels of unexplained statistical heterogeneity. ${ }^{23}$

In order to explore the sources of heterogeneity within the included studies, a subgroup analysis and meta-regression comparing the study estimates from different study-level characteristics. which will include, age, gender of the study population, the study design and quality, location of the study and sample size, ART regimes, use of statin, use of aspirin, reported measure of platelet activation (CD62P, Platelet monocyte aggregates (PMAs), sCD40, sCD62P), intervention type (type of ARV drugs used and type of antihyperglycaemic treatment) and any other important parameters identified during data extraction.

\section{Cumulative evidence}

Should there be insufficient homogeneity among the selected studies, the Cochrane collaboration tool for assessing the risk of bias ${ }^{34}$ will be used, and a qualitative summary of the included studies will be tabulated. To assess the quality and strengths of evidence across selected studies, two independent reviewers (BBN and PVD) will review the studies using the Grading of Recommendations Assessment Development and Evaluation approach. ${ }^{37}$ The approach will be implemented by the downgrading of studies based on several factors such as study limitations, indirectness of results and publication or reporting bias. The scores will be upgraded for studies with a large effect size and reported methods for adjusting of confounders. Ratings for each outcome will be categorised as high, moderate or low. This will then be followed by the rating of the overall quality. The findings will be summarised and presented in the summary of findings table.

Contributors BBN and PVD conceptualised, designed the study and drafted the protocol. ZM and TM helped draft the protocol. All authors wrote and approved the final manuscript. BBN is the guarantor of the review.

Funding This research was funded by the South Africa Medical Research Council (SAMRC). The SAMRC did not directly participate in the literature search, determination of the study eligibility, data analysis or interpretation or preparation of the manuscript for publication.

Competing interests None declared.

Provenance and peer review Not commissioned; externally peer reviewed.

Open Access This is an Open Access article distributed in accordance with the Creative Commons Attribution Non Commercial (CC BY-NC 4.0) license, which permits others to distribute, remix, adapt, build upon this work non-commercially, and license their derivative works on different terms, provided the original work is properly cited and the use is non-commercial. See: http://creativecommons.org/ licenses/by-nc/4.0/

(C) Article author(s) (or their employer(s) unless otherwise stated in the text of the article) 2017. All rights reserved. No commercial use is permitted unless otherwise expressly granted.

\section{REFERENCES}

1. Kuller LH, Tracy R, Belloso W, et al. Inflammatory and coagulation biomarkers and mortality in patients with HIV infection. PLoS Med 2008;5:e203.

2. Hanna DB, Ramaswamy C, Kaplan RC, et al. trends in cardiovascular disease mortality among persons with hiv in new york city, 20012012. Clin Infect Dis 2016;63:1122-9.

3. UNAIDS. Global AIDS Update, 2016.

4. Wang $\mathrm{H}$, Wolock TM, Carter A, et al. Estimates of global, regional, and national incidence, prevalence, and mortality of HIV, 19802015: the global burden of disease study 2015. Lancet HIV 2016;3:e361-87.

5. Rogalska-Płońska M, Rogalski P, Leszczyszyn-Pynka M, et al. Hypertension, dyslipidemia and cardiovascular risk in HIV infected adults in Poland. Kardiol Pol 2017: doi: 10.5603/KP.a2017.0148. [Epub ahead of print Jul 2017].

6. Koethe JR, Jenkins CA, Lau B, et al. Rising obesity prevalence and weight gain among adults starting antiretroviral therapy in the United States and Canada. AIDS Res Hum Retroviruses 2016;32:50-8.

7. Gresele P, Falcinelli E, Sebastiano M, et al. Endothelial and platelet function alterations in HIV-infected patients. Thromb Res 2012;129:301-8. 
8. Satchell CS, O'Halloran JA, Cotter AG, et al. Increased platelet reactivity in HIV-1-infected patients receiving abacavir-containing antiretroviral therapy. J Infect Dis 2011;204:1202-10.

9. Hruz PW. Molecular mechanisms for insulin resistance in treated HIV-infection. Best Pract Res Clin Endocrinol Metab 2011;25:459-68.

10. Ballocca F, D'Ascenzo F, Gili S, et al. Cardiovascular disease in patients with HIV. Trends Cardiovasc Med 2017;27:558-63.

11. Nkambule BB, Davison GM, Ipp H. The evaluation of platelet function in HIV infected, asymptomatic treatment-naïve individuals using flow cytometry. Thromb Res 2015;135:1131-9.

12. Holmberg SD, Moorman AC, Williamson JM, et al. Protease inhibitors and cardiovascular outcomes in patients with HIV-1. Lancet 2002:360:1747-8

13. Torres B, Guardo AC, Leal L, et al. Protease inhibitor monotherapy is associated with a higher level of monocyte activation, bacterial translocation and inflammation. J Int AIDS Soc 2014;17:19246.

14. Murata $\mathrm{H}$, Hruz PW, Mueckler M. The mechanism of insulin resistance caused by HIV protease inhibitor therapy. J Biol Chem 2000;275:20251-4

15. Vyas AK, Koster JC, Tzekov A, et al. Effects of the HIV protease inhibitor ritonavir on GLUT4 knock-out mice. J Biol Chem 2010;285:36395-400.

16. Paik IJ, Kotler DP. The prevalence and pathogenesis of diabetes mellitus in treated HIV-infection. Best Pract Res Clin Endocrinol Metab 2011;25:469-78.

17. Larson R, Capili B, Eckert-Norton M, et al. Disorders of glucose metabolism in the context of human immunodeficiency virus infection. J Am Acad Nurse Pract 2006;18:92-103.

18. Steiniche D, Jespersen S, Erikstrup C, et al. Diabetes mellitus and impaired fasting glucose in ART-naïve patients with HIV-1, HIV-2 and HIV-1/2 dual infection in Guinea-Bissau: a cross-sectional study. Trans R Soc Trop Med Hyg 2016;110:219-27.

19. Gutierrez AD, Balasubramanyam A. Dysregulation of glucose metabolism in HIV patients: epidemiology, mechanisms, and management. Endocrine 2012;41:1-10.

20. Brown TT, Tassiopoulos K, Bosch RJ, et al. Association between systemic inflammation and incident diabetes in HIV-infected patients after initiation of antiretroviral therapy. Diabetes Care 2010;33:2244-9.

21. De Wit S, Sabin CA, Weber R, et al. Incidence and risk factors for new-onset diabetes in HIV-infected patients: the Data collection on Adverse events of anti-HIV Drugs (D:A:D) study. Diabetes Care 2008;31:1224-9.
22. Stokes KY, Granger DN. Platelets: a critical link between inflammation and microvascular dysfunction. J Physiol 2012;590:1023-34

23. Schroll JB, Moustgaard R, Gøtzsche PC. Dealing with substantial heterogeneity in cochrane reviews. Cross-sectional study. BMC Med Res Methodol 2011;11:22.

24. Mayne E, Funderburg NT, Sieg SF, et al. Increased platelet and microparticle activation in HIV infection: upregulation of P-selectin and tissue factor expression. J Acquir Immune Defic Syndr 2012;59:340.

25. McCormick AL, Goodall RL, Joyce A, et al. Lack of minority K65R-resistant viral populations detected after repeated treatment interruptions of tenofovir/zidovudine and lamivudine in a resourcelimited setting. J Acquir Immune Defic Syndr 2010;54:215-6.

26. El-Sadr WM, Lundgren J, Neaton JD, et al. CD4+ countguided interruption of antiretroviral treatment. $N$ Engl J Med 2006;355:2283-96.

27. Chahroudi A, Bosinger SE, Vanderford TH, et al. Natural SIV hosts: showing AIDS the door. Science 2012;335:1188-93.

28. Matta F, Yaekoub AY, Stein PD. Human immunodeficiency virus infection and risk of venous thromboembolism. Am J Med Sci 2008;336:402-6.

29. Sauce D, Larsen M, Fastenackels S, et al. HIV disease progression despite suppression of viral replication is associated with exhaustion of lymphopoiesis. Blood 2011;117:5142-51.

30. Hunt PW, Cao HL, Muzoora C, et al. Impact of CD8+ T-cell activation on CD4+ T-cell recovery and mortality in HIV-infected Ugandans initiating antiretroviral therapy. AIDS 2011;25:2123-31.

31. McCune JM. The dynamics of CD4+ T-cell depletion in HIV disease. Nature 2001;410:974-9.

32. Moher D, Shamseer $L$, Clarke $M$, et al. Preferred reporting items for systematic review and meta-analysis protocols (PRISMA-P) 2015 statement. Syst Rev 2015;4:1.

33. Baum PD, Sullam PM, Stoddart CA, et al. Abacavir increases platelet reactivity via competitive inhibition of soluble guanylyl cyclase. AIDS 2011;25:2243-8.

34. Higgins JP, Altman DG, Gøtzsche PC, et al. The cochrane collaboration's tool for assessing risk of bias in randomised trials. BMJ 2011;343:d5928.

35. Cochran WG. The combination of estimates from different experiments. Biometrics 1954;10:101-29.

36. Higgins JP, Thompson SG. Quantifying heterogeneity in a metaanalysis. Stat Med 2002;21:1539-58

37. Balshem $\mathrm{H}$, Helfand M, Schünemann $\mathrm{HJ}$, et al. GRADE guidelines: 3 . Rating the quality of evidence. J Clin Epidemiol 2011;64:401-6. 